\title{
Determination of oil palm fruit phenolic compounds and their antioxidant activities using spectrophotometric methods.
}

\begin{abstract}
There is scarce information on the phenolics of oil palm fruits (Elaeis guineensis). In this study, phenolics were extracted from oil palm fruits and analysed using spectrophotometry for information on the different types of palm phenolics and their antioxidative activities. Analyses of the total phenolic content (TPC), total flavonoid content (TFC), o-diphenols index, hydroxycinnamic acid index, flavonols index and phenol index showed ranges between 5.64 and $83.97 \mathrm{~g} \mathrm{~L}-1$ gallic acid equivalent (GAE), 0.31-7.53 g L-1 catechin equivalent, 4.90-93.20 g L-1 GAE, 23.74-77.46 g L-1 ferulic acid equivalent, 3.62-95.33 g $\mathrm{L}-1$ rutin equivalent and 15.90-247.22 g L-1 GAE, respectively. The antioxidant assay, 2,2diphenyl-2-picrylhydrazyl radical scavenging assay, showed antioxidative activities in all the extracts with results ranging from 4.41 to $61.98 \mathrm{~g} \mathrm{~L}-1$ trolox equivalent. The high antioxidant activities of the oil palm fruit phenolics were also found to increase with increasing TPC and TFC.
\end{abstract}

Keyword: Antioxidant activities; Elaeis guineensis; Palm fruit; Phenolic compounds; Spectrophotometric indices. 